\title{
Improved Numerical Methods for Quantum Field Theory (Outstanding Junior Investigator Award)
}

\author{
Progress Report \\ for the period January 1, 1991 - December 31, 1991
}

\author{
Alan D. Sokal \\ Department of Physics \\ New York University \\ 4 Washington Place \\ New York, NY 10003 USA \\ SOKALQACF 3.NYU.EDU
}

\section{DISCLAIMER}

\begin{abstract}
This report was prepared as an account of work sponsored by an agency of the United States Government. Neither the United States Government nor any agency thereof, nor any of their employees, makes any warranty, express or implied, or assumes any legal liability or responsibility for the accuracy, completeness, or usefulness of any information, apparatus, product, or process disclosed, or represents that its use would not infringe privately owned rights. Reference herein to any specific commercial product, process, or service by trade name, trademark, manufacturer, or otherwise does not necessarily constitute or imply its endorsement, recommendation, or favoring by the United States Government or any agency thereof. The views and opinions of authors expressed herein do not necessarily state or reflect those of the United States Government or any agency thereof.
\end{abstract}

January 10, 1992

Prepared for

THE U.S. DEPARTMENT OF ENERGY

GRANT NO. DE-FG02-90ER40581 


\begin{abstract}
We are developing new and more efficient numerical methods for problems in quantum field theory. Our principal goal is to achieve rzdical re. ductions in critical slowing-down. We are concentrating at present on three new families of algorithms: multi-grid Monte Carlo (MGMC), SwendsenWang (SW) and generalized Wolff-type embedding algorithms. In addition, we are making a high-precision numerical study of the hyperscaling conjecture for the self-avoiding walk, which is closely related to the triviality problem for $\varphi^{4}$ quantum field theory.
\end{abstract}

During calendar year 1991, the principal investigator and his collaborators pursued the following investigations into new numerical methods for quantum field theory:

1) Multi-grid Monte Carlo for the two-dimensional $O(4)$-symmetric $\sigma$-model. We implemented the multi-grid Monte Carlo algorithm for the two-diriensional $O(4)$-symmetric $\sigma$-model [ $=S U(2)$ principal chiral model], including vectorization yielding 100 MFlops on a single-processor Cray Y-MP. Production runs on lattices up to $256 \times 256$ were completed in spring 1991 and analyzed during the summer. We found a dynamic critical exponent $z_{\text {int }, \mathcal{M}^{2}}=0.60 \pm 0.07$ for the Wcycle and $z_{\text {int }, \mathcal{M}^{2}}=1.13 \pm 0.11$ for the V-cycle, compared to $z_{\text {int }, \mathcal{M}^{2}}=2.0 \pm 0.15$ for the single-site heat-bath algorithm (subjective $68 \%$ confidence intervals). Thus, for this asymptotically free model, critical slowing-down is greatly reduced compared to the $z \approx 2$ of the traditional local algorithms, but not completely eliminated. (For a $256 \times 256$ lattice, W-cycle MGMC is about 35 times as efficient as a single-site heat-bath algorithm.) We are able to explain this behavior as arising from the logarithmic violations of scaling characteristic of asymptotically-free theories; but unfortunately our theory is unable to make a quantitative prediction for the dynamic critical exponent $z$. Obviously some interesting nonperturbative physics is at work here, which bears further investigation. To test our theory, a graduate scudent (Tereza Mendes) has modified our MGMC program to study the one-dimensional $O(4)$ model, which is superrenormalizable and hence has power-law (rather than logarithmic) violations of scaling. If our theory is correct, this model should exhibit dynamic critical exponent $z=0$. Production runs are planned for the near future. [Work in collaboration with Robert Edwards, Sabino Ferreira and Jonathan Goodman.] 
2) Generalized Wolff-type embedding algorithms. We have studied a class of Mon'e Carlo algorithms for the nonlinear $\sigma$-model, based on a Wolff-type embedding of Ising spins into the target manifold $M$. We have argued heuristically that such an algorithm can have dynamic critical exponent $z \ll 2$ only if the embedding is based on an involutive isometry of $M$ whose fixed-point manifold has codimension 1. Such an isometry exists only if the manifold is a discrete quotient of a product of spheres. We first tested our theory by studying the codimension- 2 algorithm for the two-dimensional $O(4)$-symmetric $\sigma$-model, for which we predict $z \approx 2$; numerical simulations yield $z_{\text {int }, \mathcal{M}^{2}}=1.5 \pm 0.5$ (subjective $68 \%$ confidence interval), in reasonable agreement with our heuristic argument. Our next test is to study the codimension- 1 algorithm for the two-dimensional $R P^{2} \sigma$-model, for which we predict $z \approx 0$ in spite of the frustration in the induced Ising model. Production runs are currently in progress, but very preliminary data indicate an exponent $z \approx 0.5$ for the "idealized" embedding algorithm and $z \approx 0.9$ for the "practical" embedding algorithm with one Swendsen-Wang hit per iteration. If these data hold up, they would indicate that our heuristic theory is qualitatively correct (codimension is important) but quantitatively incomplete ( $z$ is not simply $\approx 2$ or $\approx 0$ ). If so, interesting new nonperturbative questions are opened up. [Work in collaboration with Sergio Caracciolo, Robert Edwards and Andrea Pelissetto.]

3) Swendsen-Wang algorithms for Potts models. We are endeavoring to understand better the dynamic critical behavior of the Swendsen-Wang (SW) algorithm, which plays a key role in practical implementations of the embedding algorithms. This research has several parts:

(a) Rigorous analytical proof of lower bounds on the autocorrelation time, both for the original SW algorithm and for the Kandel et al. multi-level SW algorithm.

(b) High-precision numerical studies of the dynamic critical behavinr.

(c) Numerical and analytical investigation of the Curie-Weiss (mean-field) case.

(d) Attempts to generalize the SW algorithm to mixed ferromagnetic/antiferromagnetic Ising models. This work has imiportant applications to generalized Wolff-type embedding algorithms, as well as to spin glasses.

We succeeded in proving rigorously that a large class of multi-level SW algorithms (including the Kandel et al. algorithm) has nonzero (and in some cases quite severe) critical slowing-down, contrary to the original claims of Kandel et al.. Indeed, we showed that these algorithms obey the same lower bound $z \geq \alpha / \nu$ as the original SW algorithm. We conjecture that their dynamic critical exponent 
$z$ is equal to that of the original SW algorithm. Work on all these problems is in progress. [Work in collaboration with graduate students Xiao-Jian Li $(a, b, c)$ and Claudio Parrinello (d)].

4) Hyperscaling in the self-avoiding walk. We are employing the pivot algorithm to make a high-precision test of the hyperscaling conjecture for the selfavoiding walk [equivalent to the $N=0 O(N)$-symmetric nonlinear $\sigma$-model] in space dimensions 2,3 and 4 . This problem is closely related to the triviality problem for $\varphi^{4}$ quantum field theory. Preliminary results on the 3-dimensional case, based on walks of length up to 40000 , indicate that hyperscaling is obeyed, with a critical exponent $\nu=0.588 \pm 0.001$ and an interperetration ratio $\Psi=$ $0.248 \pm 0.001$. Both of these quantities are universal and experimentally measurable; we are currently investigating possible collaborations with polymer experimentalists. [Work in collaboration with graduate student Bin Li.]

In addition, the principal investigator delivered an invited lecture series at the Winter College on "Multilevel Techniques in Computational Physics" (International Centre for Theoretical Physics, Trieste, January-February 1991) and an invited plenary lecture at the 1991 Italian national conference on Current Problems of Theoretical Particle Physics (Isola d'Elba, May-June 1991); and he has written an invited review on Bosonic Algorithms for the book Quantum Field Theory on the Computer (edited by Michael Creutz, to be published 1992 by World Scientific).

We are in compliance with all requirements of the project agreement. Approximately $75 \%$ of the principal investigator's time since the beginning of the current term of this agreement $(7 / 15 / 90)$ has been devoted to the investigations outlined above, and we expect that a similar percentage will devoted to these investigations during the remainder of the current term.

\section{Technical reports, preprints and articles prepared for publication}

1. A.D. Sokal, How to beat critical slowing-down: 1990 update, Invited plenary lecture at the Lattice '90 conference, Nucl. Phys. B (Proc. Suppl.) 20, 55-67 (1991).

2. A.D. Sokal, Multi-grid Monte Carlo for lattice field theories, Lectures at the Winter College on "Multilevel Techniques in Computational Physics" (ICTP, Trieste, 28 January - 1 February 1991), 77 pp.

3. X.-J. Li and A.D. Sokal, Rigorous lower bound on the dynamic critical exponent of some multi-level Swendsen-Wang algorithms, Phys. Rev, Lett. 67, 1482-1485 (1991). 
4. S. Caracciolo, A. Pelissetto and A.D. Sokal, Join-and-cut algorithm for selfavoiding walks with variable length and fixed endpoints, .J. Stat. Phys. 67, no, $1 / 2$ (to appear April 1992).

5. R.G. Edwards, S.J. Ferreira, J. Goodman and A.D. Sokal, Multi-grid Monte Carlo. III. Two-dimensional $O(4)$-symmetric nonlinear $\sigma$-model, preprint (1991).

6. A.D. Sokal, Bosonic algorithms, in Quantum Field Theory on the Computer, ed. M. Creutz (Singapore: World Scientific, to appear 1992). 

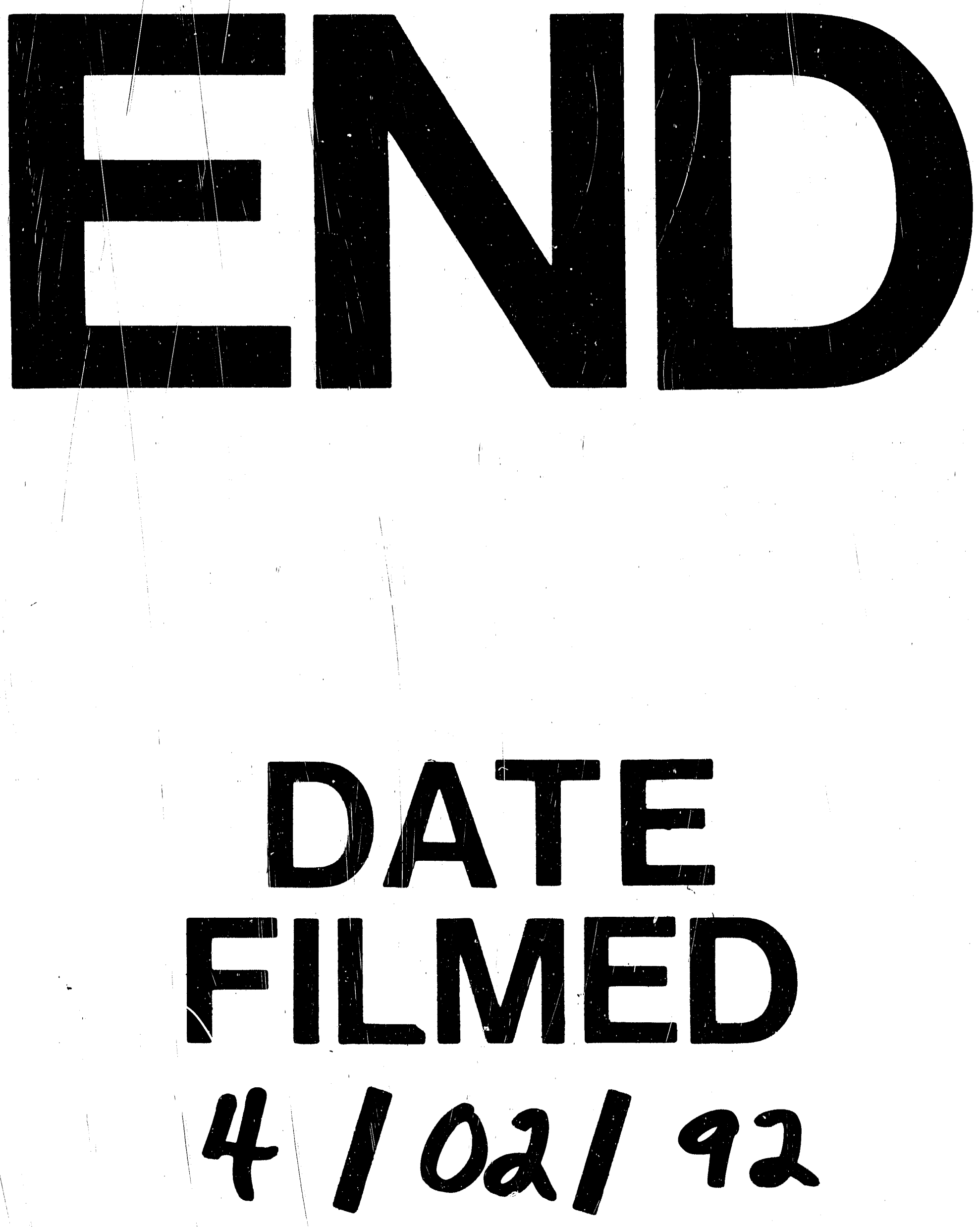


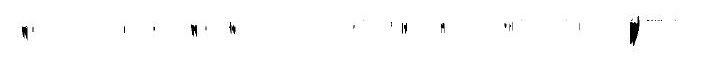

